\title{
A rare presentation of invasive mole with an arterio-venous malformation: a case report
}

\section{Atmajit Singh Dhillon, Vartika Pathak*}

Department of Obstetrics and Gynecology, Maharishi Markandeshwar Institute of Medical Sciences and Research, Mullana, Haryana, India

Received: 25 February 2020

Accepted: 27 March 2020

\section{*Correspondence:}

Dr. Vartika Pathak,

E-mail: vartika.pathak1609@gmail.com

Copyright: (c) the author(s), publisher and licensee Medip Academy. This is an open-access article distributed under the terms of the Creative Commons Attribution Non-Commercial License, which permits unrestricted non-commercial use, distribution, and reproduction in any medium, provided the original work is properly cited.

\section{ABSTRACT}

Gestational trophoblastic disease comprises of various pathologies with hydatid form mole bring a common etiology. Emergency admissions of patients of gestational trophoblastic disease is very rare. Here we present a case report of an emergency admission of patient with hydatidiform mole and severe bleeding and then was managed for the same during the course of hospital stay.

Keywords: Bleeding, Emergency, Gestational trophoblastic disease

\section{INTRODUCTION}

Trophoblastic diseases comprise of various pathological variants like molar pregnancy, invasive mole, placental site trophoblastic tumors and choriocarcinoma. Incidence of Hydatid form mole in India is 1 in 500 and the malignant potential of disease reaches $10-15 \%$ in South east Asia. ${ }^{1,2}$ Gestational trophoblastic disease is very rarely seen in the emergency department, but it must be recognized at the earliest and appropriately treated to prevent any serious complications. It can present with variety of symptoms but this disease process is very rarely documented in emergency medicine literature. ${ }^{3}$ Here we present a case of invasive mole presented to emergency with acute abdomen and severe anemia due to bleeding and was treated with salpingectomy and chemotherapy and an incidental arterio-venous malformation found on follow up was embolized as well.

\section{CASE REPORT}

A 30-year-old, female presented to emergency department to Maharishi Markhandeshwar Institute of Medical Sciences and Research (MMIMSR) Ambala, Haryana with complaints of breathlessness and abdominal distension (post-operative day 2 of exploratory laparotomy done in view) ruptured ectopic pregnancy done in a private hospital Shahjahanpur (Uttar Pradesh) on 18 March 2019. On examination, patient general condition was poor, anxious, apprehensive and tachypnea with PR-116/min BP-90/60 $\mathrm{mmHg} \quad \mathrm{RR}-32 / \mathrm{min}$ Temperature-febrile with $\mathrm{SPo} 2-78 \%$ on room air. Abdomen was distended with absent bowel sounds urgent CECT abdomen was done (Figure 1).

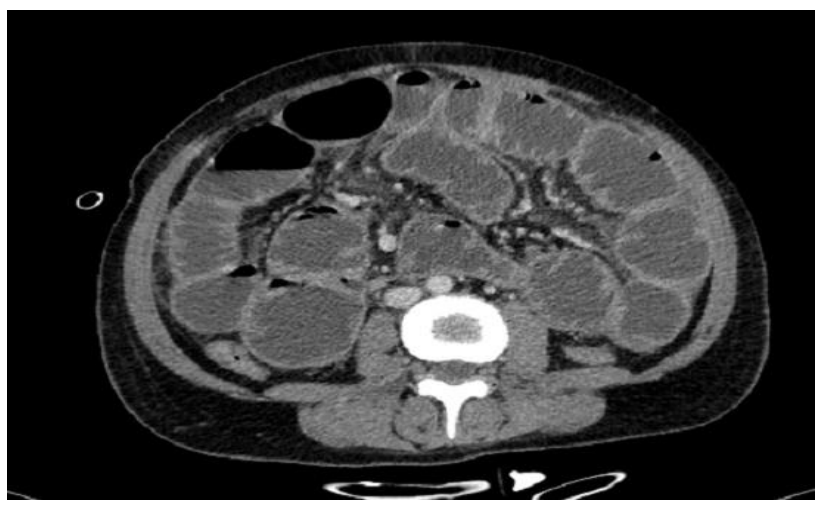

Figure 1: Dilated bowel loops likely due to paralytic ileus. No other intra-pathology detected. 
Patient was shifted to ICU and put on ventilator support. Patients relatives gave history of dilatation and evacuation 1 week before laparotomy in view of incomplete abortion, but vaginal bleeding persisted and patient condition deteriorated and she was taken up for exploratory laparotomy. Post-operatively her condition deteriorated further and was referred to study hospital. All relevant investigations were sent (hematological, biochemical, thyroid profile and chest x-ray reports were normal). Ultrasonography was done which showed large heterogeneous isoechoic mass with anechoic areas replacing myometrium of body and fundus measuring $4.4 \times 5 \mathrm{~cm}$ with marked vascularity was seen in the mass suggestive of gestational trophoblastic neoplasia. She further underwent CT scan whole abdomen and pelvis which was suggestive of invasive mole. S. Beta HCG levels were sent which came out to be 81000 units. Since no documentation was available with the patient relatives, operating surgeon was called and it was informed that there was a perforation at the level of fundus in midline from where products of conceptus were removed and sent for histopathology examination Figure 2.

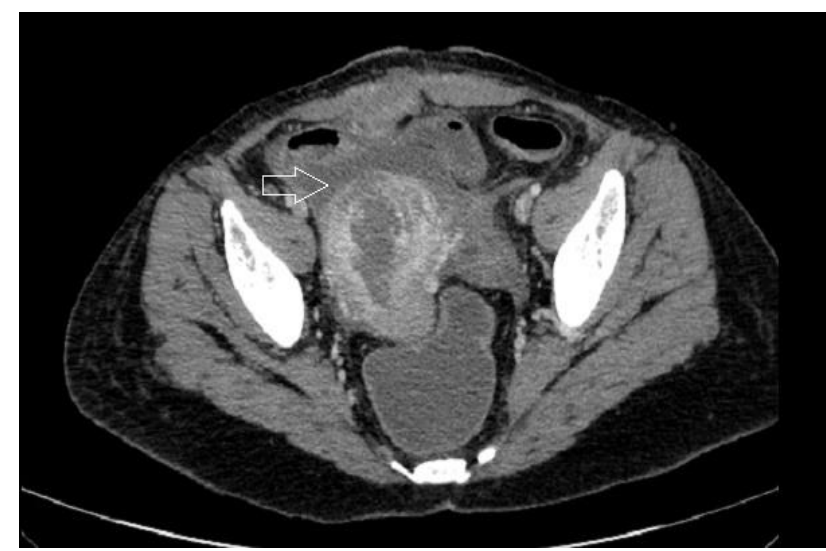

Figure 2: AXIAL CECT at the level of pelvis showing rent at the level of fundus in the uterus with irregular contour with echogenic content seen in the peri uterine location suggestive of hematoma.

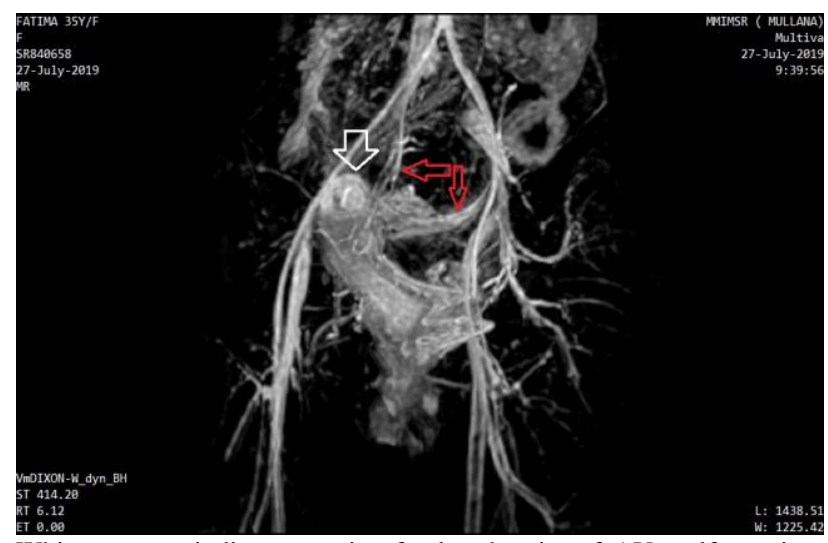

White arrow - indicates uterine fundus the site of AV malformation Red arrow- indicates feeding vessels arising from internal iliac.

Figure 3: CEMRI angiography on coronal section (contrast enhance MRI).
Histopathology report suggestive of trophoblastic tissue. Based on the collective findings, diagnosis of invasive mole was made. Patient was started on chemotherapy with methotrexate, after baseline investigations were done (liver function tests, renal function tests, complete blood count, thyroid function test and chest $\mathrm{x}$-ray) was done which came out to be normal. After 4 cycles of chemotherapy beta HCG levels fall down to 2.45 units and patient was given 2 more cycles of chemotherapy. Ultrasonography was repeated after each chemotherapy cycles which showed regression in the size of uterine mass. After 6 cycles of chemotherapy, when transvaginal sonography scan done in gynecology OPD in MMIMSR it was found that the invasive lesions in myometrium had regressed. However, there was localized area of vascularity seen in the fundus of uterus and AV malformation was suspected which was further confirmed in MRI angiography Figure 3.

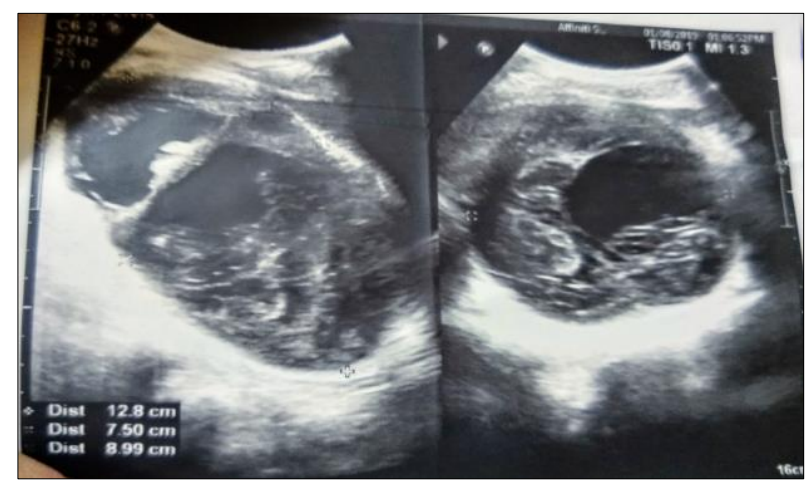

Figure 4: Ultrasound abdomen of large collection in the left adnexa measuring $12.8 \times 9 \times 7.5 \mathrm{~cm}$ seen in the left adnexa with embedded left ovary.

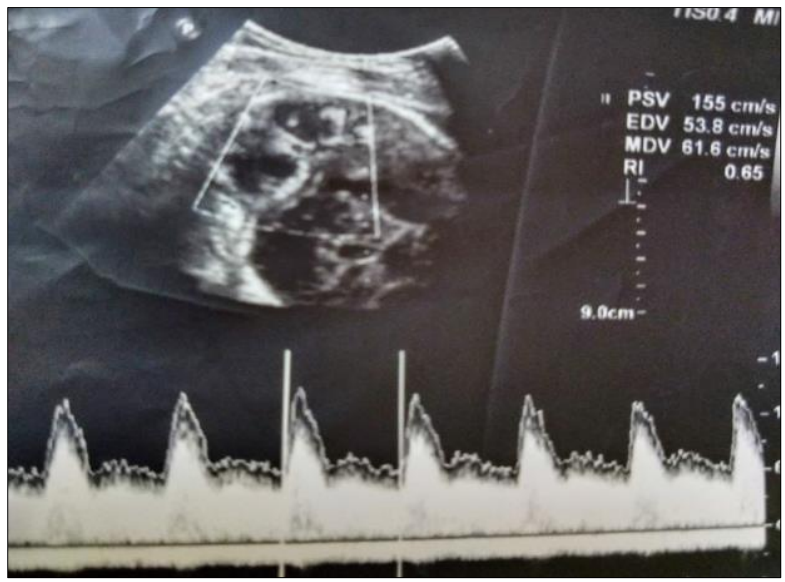

Figure 5: Color Doppler of lesion at the level of fundus of uterus with flow on color Doppler (PSV $155 \mathrm{~cm} / \mathrm{sec}$ ) giving a confirmation of AV malformation.

Patients relatives were counselled for embolization but they did not pay heed to the advice. After 7 days of last chemotherapy she presented in Emergency department of Maharishi Markandeshwar Institute of Medical science 
and research with acute pain abdomen, general condition stable vital parameters normal and on ultrasonography AV malformation and left adnexal mass, was detected and it was suspected that there was sustained low volume blood leaking from AV malformation and collected around left ovary and resulting in the resultant hematoma around ovary, presenting as left ovarian mass Figure 4, Figure 5 .

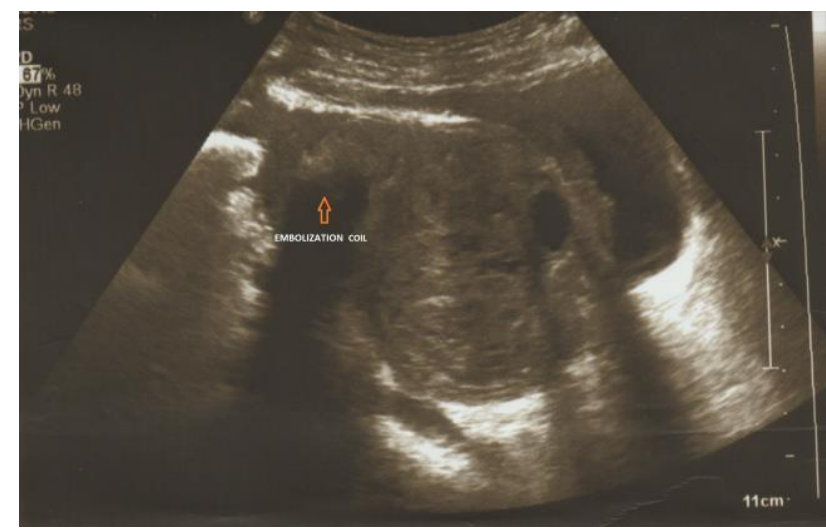

Figure 6: Embolization coil seen in situ with residual collection in the left adnexa.

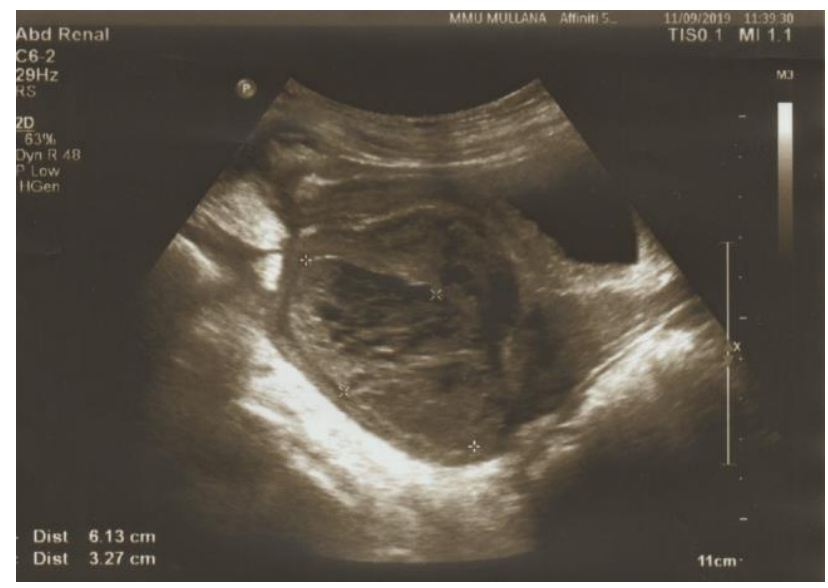

Figure 7: Residual collection in the left adnexa measuring $6.1 \times 3.3 \mathrm{~cm}$.

On examination, general condition stable, vitals were stable but abdominal tenderness was present. Pelvic examination revealed a bulky uterus with left adnexal mass $6 \times 6 \mathrm{~cm}$ irregular and decreased mobility. Patient subsequently underwent uterine artery embolization, and thereafter patient was asymptomatic. On follow up visits, there was resolution of the vascular malformation with no vascularity seen on subsequent repeated transvaginal sonography. Patient remained asymptomatic the left adnexal mass resolved; serum beta HCG levels were normal on follow up visits Figure 6, Figure 7.

\section{DISCUSSION}

Gestational trophoblastic neoplasms most commonly develop after complete Hydatid form mole pregnancy and can also develop after live birth, miscarriage or termination of pregnancy. ${ }^{4}$

Kumar et al, reported a case of invasive mole presenting as acute hemoperitoneum as the molar tissue can penetrate myometrium and can lead to uterine perforation and cause vaginal bleeding due to erosion of uterine vessels. ${ }^{5}$ There was a misdiagnosis in the case prior to hospitalization in our institute when incomplete abortion was diagnosed dilatation and evacuation was done and subsequently laparotomy was done without proper evaluation of the patient.

Diagnostic imaging may begin with an initial ultrasound of the pelvis, but CT of the abdomen and pelvis is recommended to evaluate for the extent of the disease. It is currently recommended that monthly HCG levels are checked for 6 months after the level has returned to normal because of the risk of persistent disease. GTN should be suspected and further investigated in the setting of a preceding molar pregnancy when post-evacuation HCG levels plateau or rise. ${ }^{6,7}$

Invasive mole is highly sensitive to chemotherapy. Combination chemotherapy should be continued in such cases as far as toxicity permits until the patient achieves 3 consecutive normal HCG levels. As normal HCG levels are attained, 3 additional courses of chemotherapy are administered to reduce the risk of relapse. ${ }^{8,9}$ The differential diagnosis of intramural vascular lesions of the myometrium includes arteriovenous malformation, gestational trophoblastic neoplasia, hemangiomata, sarcoma and interstitial pregnancy.

The causes of Arteriovenous malformation (AVM) can be either congenital or acquired. Acquired AVM are reported as a consequence of previous uterine trauma such as curettage procedure, cesarean section or pelvic surgery and it is also associated with infection, retained products of conception, gestational trophoblastic disease and malignancy. Serum beta HCG levels can distinguish pregnancy related conditions from uterine AVM pelvic ultrasound color Doppler and spectral analysis along with MRI angiography can aid in the diagnosis of condition and reduce the gap between diagnosis and treatment. So, in all these cases high index suspicion for AVM in the uterus should be there. In this case patient developed a AVM which was promptly diagnosed and treated. The diagnosis of uterine $\mathrm{AV}$ malformation is challenging given the rarity of the condition and non-specific ultrasound findings. In this particular case, on follow up ultrasound post chemotherapy raised the suspicion of arteriovenous malformation which was subsequently confirmed and treated.

\section{CONCLUSION}

At times, emergency physician may have to deal with post-partum vaginal bleeding or unexplained vaginal bleeding and associated unexplained uterine or adnexal 
enlargement. It is important to be aware of the rare features of gestational trophoblastic neoplasms and be able to recognize the patient with this potentially lethal disease. All cases of pain or bleeding per vaginum should be evaluated with a high-resolution ultrasound and serum beta HCG assay and the possibility of trophoblastic disease should be entertained in the patients of the reproductive age group.

Funding: No funding sources

Conflict of interest: None declared

Ethical approval: Not required

\section{REFERENCES}

1. Muggia FM, Eifel PJ, Burke TW. Gestational trophoblastic diseases. In: de Vita VJ Jr, Hellman S, Rosenberg SA, editor. In Cancer Principles and Practice of Oncology. 5. Lippincott-Raven, Philadelphia; 1997:1427-1539.

2. Balagopal $\mathrm{P}$, Pandey $\mathrm{M}$, Chandramohan $\mathrm{K}$, Somanathan T, Kumar A. Unusual presentation of choriocarcinoma. World J Surg Oncol. 2003;1(1):4.

3. Bruner DI, Pritchard AM, Clarke J. Uterine rupture due to invasive metastatic gestational trophoblastic neoplasm. West J Emerg Med. 2013;14(5):444-7.

4. Seckl MJ, Sebire NJ, Berkowitz RS. Gestational trophoblastic disease. Lancet. 2010;376:717-29.
5. Aminimoghaddam S, Maghsoudnia A. Unusual presentation of invasive mole: a case report. J Reprod Infertil. 2017;18(1):205-9.

6. Garner EI, Garrett A, Goldstein DP. Significance of chest computed tomography findings in the evaluation and treatment of persistent gestational trophoblastic neoplasia. J Reprod Med. 2004;49:4119.

7. Kohorn EI. The new FIGO 2000 staging and risk factor scoring system for gestational trophoblastic disease: Description and critical assessment. Inter $\mathbf{J}$ Gynecol Cancer. 2001;11:73-7.

8. Tidy J, Hancock BW. The management of gestational trophoblastic disease. RCOG Green-top Guideline No.: 38; UK: Royal College of Obstetricians and Gynaecologists. 2010:11.

9. Berkowitz RS, Goldstein DP. Current advances in the management of gestational trophoblastic disease. Gynecol Oncol. 2013;128(1):3-5.

Cite this article as: Dhillon AS, Pathak V. A rare presentation of invasive mole with an arterio-venous malformation: a case report. Int J Reprod Contracept Obstet Gynecol 2020;9:2180-3. 